\title{
Rendimiento académico, enfoques de trabajo e implicación en los deberes escolares
}

Academic Performance, Work Approaches and Homework Involvement Rendement académique, perspectives de travail et implication dans les devoirs scolaires

Rendimento acadêmico, enfoques de trabalho e implicação nos deveres de casa

Fecha de recepción: 20 DE FEBRERO DE 2016/Fecha de aceptación: 25 DE JULIO DE 2017/Fecha de disponibilidad en línea: 1 DE DICIEMBRE DE 2017

Encuentre este artículo en http://magisinvestigacioneducacion.javeriana.edu.co/

doi: 10.11144/Javeriana.m10-20.raet

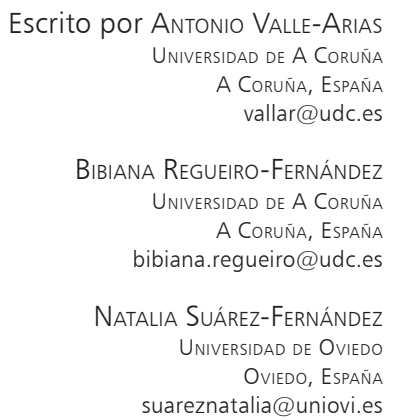
José C. NúÑez-PÉREZ UnIVERSIDAD DE OVIEDO Oviedo, España jcarlosn@uniovi.es
Pedro Rosário UnIVERSIDAD DE Minho Braga, Portugal prosario@psi.uminho.pt
IRENE PAN-LÓPEZ UnIVERSIDAD DE A CORUÑA A Coruña, España irenepan@edu.xunta.es

\begin{abstract}
Resumen
El objetivo de este artículo de investigación fue analizar la relación entre enfoque de trabajo, implicación en los deberes escolares y rendimiento académico en matemáticas. Participaron 897 estudiantes de $5^{\circ}$ y $6^{\circ}$ curso de Educación Primaria $(50,2 \%$ chicos y $49,8 \%$ chicas). Los resultados indican que a mayor rendimiento académico mayor enfoque profundo en la realización de los deberes escolares, mayor cantidad de deberes realizados y mejor aprovechamiento del tiempo. No obstante, el tiempo que los estudiantes dedican a realizar los deberes es mayor cuando el rendimiento es bajo o medio. Se discuten implicaciones educativas.
\end{abstract}

\section{Palabras clave}

Deberes escolares; procesos de estudio; implicación;

matemáticas; enseñanza primaria

Para citar este artículo / To cite this article / Pour citer cet article / Para citar este artigo

Valle, Antonio; Regueiro, Bibiana; Suárez, Natalia; Núñez, José C.; Rosário, Pedro \& Pan, Irene (2017). Rendimiento académico, enfoques de trabajo e implicación en los deberes escolares. magis, Revista Internacional de Investigación en Educación, 10 (20), 123-142. https://doi.org/10.11144/ Javeriana.m10-20.raet 
Keywords

Homework; study processes;

involvement; mathematics:

primary education

\section{Mots clés}

Devoirs scolaires; processus d'étude; implication; mathématiques; enseignement primaire

\section{Abstract}

The main objective of this article was to analyze the relationship between work approach, homework involvement and academic performance in mathematics. 897 students from $5^{\text {th }}$ and $6^{\text {th }}$ grades participated ( $50.2 \%$ boys and $49.8 \%$ girls). Results indicate that the higher the academic achievement, the greater the focus on doing a homework, the greater the number of homeworks done and the better use of time. However, the time students spend doing a homework is greater when their academic performance is low or medium. Educational implications are discussed.

\section{Résumé}

L'objectif de cet article de recherche a été faire l'analyse du rapport entre la perspective du travail, implication dans les devoirs scolaires et le rendement académique en mathématiques. 897 étudiants de $5^{\text {ème } y}$ $6^{\text {ème }}$ course d'éducation primaire ont participés (50,2\% garçons y 49,8\% filles). Les résultats indiquent que le rendement académique est plus grand lorsque la concentration dans la réalisation des devoirs scolaires est plus importante et approfondie, lorsqu'il y a une plus grande quantité de devoirs faits il y a un meilleur profit du temps. Néanmoins, le temps que les étudiants dédient à faire les devoirs est plus grand lorsque le rendement est bas ou moyen. On discute les implications éducatives.

\section{Palavras-chave}

Deveres escolares, processos de estudo, envolvimento, matemáticas, ensino primário

\section{Resumo}

O objetivo deste artigo de pesquisa foi analisar a relação entre enfoque de trabalho, implicação nos deveres de casa e rendimento acadêmico em matemáticas. Participaram 897 estudantes de $5^{\circ}$ e $6^{\circ}$ curso de educação primária $(50,2 \%$ meninos e $49,8 \%$ meninas). Os resultados indicam que a maior rendimento acadêmico maior enfoque profundo na realização dos deveres de casa, maior quantidade de deveres realizados e melhor aproveitamento do tempo. Não obstante, o tempo que dedicam os estudantes a realizar os deveres é maior quando o rendimento é baixo ou médio. Discutem-se implicações educativas. 


\section{Introducción}

Numerosos informes internacionales, como los elaborados por la International Association for the Evaluation of Educational Achievement (IEA, 2011) o la Organización para la Cooperación y el Desarrollo EconómiCOs, OCDE (MECD, 2013), resultan muy clarificadores a la hora de señalar el bajo rendimiento en matemáticas obtenido por los escolares españoles en comparación con los de otros países y dentro de nuestro país con respecto a otras materias del currículo.

Según varias pruebas de evaluación internacionales, como la TIMSS (Trends in International Mathematics and Science Study), realizada por la IEA (2011) y en la que participaron 4.183 alumnos de $4^{\circ}$ de Primaria, el alumnado español obtiene un total de 482 puntos (mientras el promedio de los países participantes es de 491 puntos), lo cual lo sitúa a la cola de los 63 países evaluados. La proporción de alumnos con bajo rendimiento en matemáticas es de un $13 \%$ frente al $7 \%$ de la media OCDE, mientras que la proporción de alumnos excelentes en esta área resulta de tan solo $1 \%$, frente al 5\% de la media OCDE. En una situación similar nos deja el informe PISA, realizado por la OCDE (2013), el cual señala a Shangai-China (613 puntos), Singapur (573 puntos) y Hong-Kong-China (561 puntos) en los puestos más altos del ranking, relega a España, por su puntuación media de 484 puntos, al puesto 34 de los 66 evaluados y la sitúa a la cola en rendimiento matemático respecto al grupo de los países europeos y en la media de los países OCDE.

Sin duda, las razones de estos malos resultados hay que buscarlas en una confluencia de factores que incluyen aspectos relacionados con la baja calidad de los procesos de enseñanza de esta materia, las dificultades asociadas a los propios contenidos matemáticos, la falta de motivación del alumnado, la escasa utilidad percibida por parte de los estudiantes de los contenidos matemáticos que se les enseñan, etc. Además, habría que tener en cuenta también el posible efecto de otros factores contextuales (por ejemplo, el centro escolar), como han puesto de manifiesto algunos estudios (ver p.e., Woitschach, Fernández-Alonso, Martínez-Arias \& Muñiz, 2017).

Como en el resto de materias académicas, una parte muy importante de los aprendizajes matemáticos se llevan a cabo dentro del aula, donde el profesorado encargado de la docencia de esta materia transmite sus contenidos al grupo de estudiantes. Pero también es verdad que, del mismo modo que acontece en otras asignaturas, otra parte importante de los aprendizajes tiene lugar fuera del aula y sin la presencia física del profesorado de la materia. Se trata de aquellas tareas y actividades que los estudiantes desarrollan fuera del horario escolar, por lo general en el hogar. Aunque esta definición puede parecer un tanto simple, la mayor parte de los autores coincide en señalar que los deberes escolares son una compleja herramienta educativa, dado el gran número de factores que influyen en ella y la diversidad de actores y contextos implicados (Cooper, 2001; Corno, 2000; Warton, 2001). Harris Cooper (2001) define en un principio los deberes escolares como las tareas que los profesores prescriben a los alumnos para realizar fuera del horario lectivo y que tienen como finalidad consolidar, fortalecer y ampliar conocimientos ya trabajados dentro del aula. Posteriormente, Harris Cooper, Jorgianne Civey Robinson y Erika A. Patall (2006) amplían su concepción sobre los deberes escolares y las contemplan no como una simple tarea académica, sino como un proceso complejo y dinámico que trasciende las fronteras de la escuela y se asienta en el ambiente familiar.
Descripción del artículo | Article description | Description de l'article | Artigo descrição

Este artículo de investigación es parte de la investigación más amplia Relación entre deberes escolares y rendimiento académico: una perspectiva multinivel y longitudinal, desarrollada en el marco del proyecto de investigación EDU2013-44062-P, perteneciente al Plan Estatal de Investigación Científica y Técnica y de Innovación 2013-2016 (Ministerio de Economía, Industria y Competitividad, MINECO) y al financiamiento recibido por una de las autoras en el programa Formación de Profesorado Universitario, FPU del Ministerio de Educación, Cultura y Deporte, MECD. 
Aunque no hay unanimidad sobre si hacer deberes es positivo, negativo o neutro para el aprendizaje y el rendimiento académico, dado que las tareas para casa o los deberes escolares que realizan los estudiantes fuera del aula son un elemento más del proceso de aprendizaje de las matemáticas, parece interesante conocer el papel que desempeñan en dicho proceso y analizar las relaciones entre el rendimiento académico en matemáticas, los enfoques de trabajo que adopta el estudiante al hacer los deberes escolares en esta asignatura y su grado de implicación al realizarlos.

Enfoques de trabajo al realizar los deberes escolares

En la investigación sobre los deberes escolares es fundamental profundizar en los aspectos motivacionales y cognitivos del estudiante y conocer con mayor detalle cómo es el proceso de realización de esos deberes. Por ello, el enfoque de trabajo que adopta el estudiante cuando se enfrenta a las tareas es uno de los aspectos que mayor información nos puede aportar respecto a los motivos y metas que pretende conseguir al hacer los deberes y también sobre las estrategias que pone en marcha para tratar de dar una respuesta lo más ajustada posible a esas metas y motivos.

Con base en las propuestas planteadas hace años por algunos autores (p.e., Biggs, 1988) en las que el enfoque de aprendizaje se concebía como la forma en que un alumno se aproxima a sus tareas académicas y las afronta poniendo en marcha determinadas estrategias de estudio, influidas por la motivación y la forma de concebir el aprendizaje, se considera que el enfoque de trabajo que emplea el estudiante al hacer los deberes escolares sigue unas pautas idénticas en sus aspectos motivacional y cognitivo.

Así, se han diferenciado dos grandes enfoques de aprendizaje como definitorios de modos distintos de enfrentarse a las tareas académicas: el enfoque profundo y el enfoque superficial. Según John B. Biggs (1987), los alumnos que adoptan un enfoque profundo se caracterizan por que ponen interés en la tarea académica y disfrutan llevarla a cabo, buscan el significado inherente a la misma, la personalizan, la hacen significativa para la propia experiencia y el mundo real, integran aspectos o partes de la tarea en un todo, ven relaciones entre ese todo y el conocimiento previo, intentan teorizar acerca de la tarea, y conforman hipótesis. Estos estudiantes se caracterizan por su interés en alcanzar una comprensión significativa de los contenidos de la materia, y para ello adoptan estrategias que los lleven a comprender el significado inherente de esta, e intentan relacionarla con sus conocimientos previos (Doménech-Betoret \& Gómez-Artiga, 2011).

Por el contrario, el alumnado que adopta un enfoque superficial concibe la tarea como una demanda para cumplir, una imposición necesaria para alcanzar otras metas; ve los aspectos o partes de la tarea como no relacionados entre sí, se preocupa por la cantidad de tiempo que lleva completar esta tarea, evita significados personales o de otro tipo que la tarea pueda implicar y, por último, depende de la memorización e intenta reproducir sus aspectos superficiales.

En un buen número de estudios, el enfoque superficial está relacionado con bajos resultados académicos (Rosário, González-Pienda, Cerezo, Pinto, Ferreira, Abilio \& Paiva, 2010). Por el contrario, los alumnos que utilizan un enfoque profundo en su trabajo escolar están intrínsecamente motivados y utilizan estrategias que maximizan la comprensión de los contenidos que pretenden aprender. La utilización persistente de este enfoque en el trabajo personal se asocia con niveles elevados de comprensión y de rendimiento, siempre dependiendo de los criterios de evaluación, pues algunos profesores valoran el detalle más que la justificación comprehensiva de los razonamientos (Biggs, 1993). 
Héfer Bembenutty y Marie Catherine White (2013) afirman que cuando los estudiantes realizan los deberes escolares con un enfoque profundo y muestran interés en la tarea y una actitud positiva, suelen alcanzar un buen rendimiento académico en las distintas materias. Además, destacan que cuando los procesos autorregulatorios se integran como parte de la realización de los deberes escolares, los alumnos obtienen experiencias de aprendizaje positivas y motivadoras. Por otro lado, Marie Catherine White (2011) considera que las percepciones de autoeficacia, la motivación intrínseca en la tarea y la búsqueda de ayuda en los deberes se relacionan positivamente con los resultados académicos. Por tanto, el interés en la tarea, la actitud positiva y la adopción de un enfoque de trabajo profundo contribuyen a mejorar el rendimiento en las distintas materias.

Jianzhong Xu y Hongyun Wu (2013) establecen una relación positiva entre la gestión de los deberes escolares y su realización por medio de un enfoque profundo, con la estabilidad emocional, la ayuda familiar en las tareas, el interés por las mismas, así como el feedback proporcionado por el profesorado y el uso de estrategias de autorregulación del aprendizaje. Por otro lado, José Carlos Núñez, Guillermo Vallejo, Pedro Rosário, Ellián Tuero y Antonio Valle (2014) encontraron que cuanto más utilizaba el alumno un enfoque profundo de estudio y aprendizaje, mayor era su rendimiento académico y cuanto más superficial era el enfoque, menor era el rendimiento académico.

En su vertiente práctica, la metáfora superficial versus profundo constituye un marco conceptual rápidamente perceptible tanto en el contexto de clase como en los demás contextos educativos (p.e., realización de los deberes escolares en el hogar), y cualitativa y cuantitativamente, ha demostrado ser una poderosa herramienta para que los padres, profesores y alumnos conceptualicen las tareas académicas (Biggs, 1993). El núcleo sustantivo del concepto de enfoques de estudio (o aprendizaje) es la conexión metacognitiva entre una intención para abordar una tarea y una estrategia para ponerla en marcha (Rosário, Núñez, Ferrando, Paiva, Lourenço, Cerezo \& Valle, 2013).

En el contexto del hogar, el proceso de realización de los deberes se centra en lo que hace el estudiante al enfrentarse a los deberes, es decir, en cómo aborda su trabajo y en cómo gestiona los recursos personales y ambientales cuando hace los deberes. Todo parece indicar que el enfoque de trabajo empleado al hacer los deberes incide no solo en la ejecución final de esos deberes, sino también en la calidad de su proceso de realización.

Aquellos que adoptan un enfoque más profundo realizarán los deberes y se implicarán en ellos con la intención de aprender y reforzar los conocimientos adquiridos en clase, y se preocuparán por solventar las dudas que se les plantean durante su realización y relacionar los deberes con lo aprendido previamente (Valle, Pan, Regueiro, Suárez, Tuero \& Nunes, 2015). Implica, por tanto, un propósito intrínseco de comprender las ideas y usar estrategias para crear significado. Por el contrario, si los estudiantes ponen en marcha un enfoque poco profundo (superficial) realizarán los deberes porque se sienten obligados por el profesor y tal vez por sus padres; su objetivo será terminarlos cuanto antes para poder dedicarse a otras actividades más divertidas y solo se preocuparán de llevarlos hechos porque hay que entregarlos y corregirlos en clase, pero no porque piensen sacar provecho de su realización para avanzar en sus aprendizajes.

Por tanto, el enfoque de trabajo que pone en marcha el estudiante al hacer los deberes condiciona no solo el proceso y los resultados de este, sino también su grado de implicación al enfrentarse a esos deberes. 
Implicación de los estudiantes en los deberes escolares

La mayor parte de la investigación incluye tres variables relevantes vinculadas con la implicación de los estudiantes en los deberes escolares: la cantidad de deberes prescritos por el profesorado que realizan los alumnos, el tiempo dedicado a hacer los deberes y el aprovechamiento del tiempo que les dedican (Regueiro, Rodríguez, Piñeiro, Estévez, Ferradás \& Suárez, 2015; Valle, Regueiro, Estévez, Piñeiro, Rodríguez \& Freire, 2015). Estas tres variables delimitan la implicación o compromiso conductual (behavioral engagement) de los estudiantes en los deberes escolares (Fredricks, Blumenfeld \& Paris, 2004).

Cantidad de deberes escolares realizados

Hay un gran debate sobre cómo el rendimiento académico de los estudiantes se ve afectado por la cantidad de deberes realizados y el tiempo dedicado a su ejecución. En la investigación llevada a cabo por Harris Cooper, James J. Lindsay, Barbara Nye y Scott Greathouse (1998) se comprobó que si bien la cantidad de deberes que los profesores prescriben es importante, el porcentaje de deberes que los alumnos realizan resulta más importante. En todo caso, debe tenerse muy en cuenta a qué nos referimos exactamente cuando hablamos de cantidad, pues la mayoría de los estudios utilizan este término de forma indistinta para referirse a la cantidad de deberes asignados por el profesor y a la cantidad de deberes que completan los alumnos (Cooper, Lindsay, Nye \& Greathouse, 1998).

Parece demostrado que los estudiantes que completan sus deberes escolares tienen mejores calificaciones académicas que aquellos que no los completan (Cooper, 1989b; Trautwein, Köller, Schmitz \& Baumert, 2002). Por tanto, la cantidad de deberes realizados por los estudiantes, de los que prescribe el profesor, suele relacionarse positivamente con una mejora en su rendimiento académico. De hecho, Harris Cooper, James J. Lindsay, Barbara Nye y Scott Greathouse (1998) comprobaron que la cantidad de deberes que realizan los alumnos se correlaciona positivamente con su rendimiento académico, tanto en los alumnos de cursos avanzados como en alumnos de cursos inferiores.

Un gran número de trabajos constata que de todas las variables relacionadas con la implicación en los deberes escolares, la cantidad de deberes que los alumnos realizan (de los prescritos por el profesorado) parece ser la variable que mejor predice el rendimiento académico (Valle, Pan, Núñez, Rosário, Rodríguez \& Regueiro, 2015). En la misma línea, Joyce L. Epstein y Frances L. van Voorhis (2001) indican que un mayor número de deberes y una mejor disciplina serían dos argumentos de gran importancia para generar un ambiente de aprendizaje adecuado y conseguir el éxito académico.

Tiempo dedicado a realizar los deberes escolares

Una parte muy importante de la investigación sobre los deberes escolares ha estado muy centrada en estudiar cómo el tiempo empleado por los alumnos en la realización de los mismos se relaciona con el rendimiento académico (Valle, Regueiro, Estévez, Piñeiro, Rodríguez \& Freire, 2015).

El tiempo dedicado al estudio y a la realización de los deberes suele utilizarse como uno de los indicadores de éxito académico, hasta tal punto que siempre se ha tratado de concienciar a los alumnos de que emplear más tiempo en los mismos les proporcionará mejores resultados académicos. En general, muchos de los trabajos confirman que un incremento en el tiempo empleado por los alumnos, en especial en los cursos más elevados, tiene un efecto positivo (Keith, 1982). A pesar de que algunos autores con- 
sideran que emplear más tiempo en los deberes tiene efectos positivos en los logros académicos (Olympia, Sheridan, Jenson \& Andrews, 1994) y de que muchas investigaciones hallan evidencia empírica de una relación significativa y positiva entre tiempo dedicado a los deberes y rendimiento, los resultados de otras investigaciones más recientes parecen discutir esta relación (Núñez, 2012).

En todo caso, parece evidente que los resultados no aportan datos concluyentes en torno a la relación entre tiempo dedicado a los deberes y rendimiento académico de los estudiantes. De hecho, mientras algunos estudios encuentra una relación positiva (Cooper, Jackson, Nye \& Lindsay, 2001; Cooper, Robinson \& Patall, 2006; Trautwein, Köller, Schmitz \& Baumert, 2002; Trautwein \& Lüdtke, 2009) otros hallan una relación débil e incluso negativa (De Jong, Westerhof \& Creemers, 2000; Tam, 2009; Trautwein, 2007; Trautwein, Köller, Schmitz \& Baumert, 2002; Trautwein, Lüdtke, Schnyder \& Niggli, 2006; Trautwein, Schnyder, Niggli, Neumann \& Lüdtke, 2009).

Incluso esta relación varía según la edad del estudiante: de modesta a débil entre los estudiantes en los cursos altos (p.e., Cooper \& Valentine, 2001) y ausencia de relación en el caso de los estudiantes de primaria (Cooper, Lindsay, Nye \& Greathouse, 1998). Una de las razones por las cuales la relación entre deberes y rendimiento es más débil en primaria que en secundaria puede ser que la asignación de deberes que hacen los profesores en los primeros cursos suele tener como objetivo prioritario que los alumnos aprendan a gestionar mejor su tiempo mediante una revisión del material de clase, mientras que el profesorado de secundaria asigna esos deberes para enriquecer y perfeccionar los conocimientos impartidos en clase (Muhlenbruck, Cooper, Nye \& Lindsay, 2000).

\section{Aprovechamiento del tiempo dedicado a los deberes y rendimiento académico}

Cuando se habla de tiempo dedicado a los deberes, la investigación recomienda establecer una diferenciación clara entre la cantidad y la calidad de este tiempo. Las correlaciones positivas entre tiempo empleado en los deberes y rendimiento no deben ser tomadas como una evidencia de que, por sí solo, más tiempo empleado en los deberes conduce necesariamente a un elevado rendimiento, ya que dedicar demasiado tiempo a los deberes puede significar insuficientes conocimientos previos o dificultades de aprendizaje para trabajar los contenidos. Por lo tanto, más que el tiempo en sí mismo, hay que tener muy presentes el proceso y el aprovechamiento real de ese tiempo cuando los estudiantes realizan los deberes escolares.
El esfuerzo invertido en los deberes ha demostrado tener un impacto más positivo en el rendimiento académico que el tiempo dedicado a los deberes (Trautwein, Lüdtke, Kastens \& Köller, 2006), ya que el esfuerzo que un alumno emplea en la realización de los deberes escolares no tiene por qué estar necesariamente relacionado con el tiempo que tarda en realizarlos (Regueiro, Pan, Valle, Núñez, Suárez \& Rosário, 2014). Así, se indica que para comprender mejor lo que predice el mayor o menor esfuerzo en los deberes en materias diferentes es necesario analizar si los procesos relacionados con un elevado esfuerzo son los mismos en los diferentes dominios, y se habla por tanto más en términos de calidad que de cantidad de tiempo empleado.

Por estos motivos, el estudio de la relación entre el tiempo invertido en los deberes y el rendimiento académico debería incluir otros aspectos relativos al proceso de realización de los deberes (Rosário, Mourão, Baldaque, Nunes, Núñez, González-Pienda, Cerezo \& Valle, 2009) y contemplar otras variables quizás más relevantes en este proceso como, por ejemplo, todas aquellas relacionadas con las habilidades de gestión del tiempo dedicado a realizar los deberes. De este modo, lo más probable es que la relación entre la cantidad de deberes realizados, el tiempo dedicado a los mismos y el rendimiento académico pueda estar mediatizada por el aprovechamiento real del tiempo empleado por el estudiante al realizar los deberes.

\section{Deberes escolares y rendimiento académico}

En los primeros estudios sobre los deberes escolares, los investigadores simplemente comparaban el rendimiento de alumnos a los que se les asignaban estas tareas con el rendimiento de otros que no tenían que hacer esas tareas ni otras que ocuparan su tiempo libre en casa. Harris Cooper (1989a) encontró 20 estudios de este tipo, de los cuales 14 mostraban resultados a favor de los deberes y 6 mostraban resultados en contra. Se observó una clara influencia del curso al que los alumnos asistían en la efectividad de los deberes.

J. Barry McGrath (1992) estudió el efecto de los deberes en el rendimiento en 94 institutos de secundaria en tres clases de inglés en cada uno de los centros. La mitad de los estudiantes no tenía que hacer deberes y la otra mitad, sí. La asignación de los estudiantes a cada condición se realizó al azar. El experimento duró tres semanas e implicaba 12 tareas para casa. Los estudiantes que hicieron los deberes obtuvieron resultados significativamente mejores en el postest de rendimiento. Estudios llevados a cabo por Harvey Charles Foyle (1984, 1990) también obtuvieron resultados en concordancia con lo anterior: los alumnos que fueron asignados a la condición aleatoria de hacer deberes obtuvieron mejores resultados en un postest 
que los asignados a la condición sin deberes. Ulrich Trautwein, Olaf Köller, Bernhard Schmitz y Jürgen Baumert (2002) concluyen tras una investigación que los deberes están muy relacionados con el rendimiento en matemáticas. Aunque la frecuencia de los deberes tiene efectos positivos, las tareas cortas mostraron ser tan buenas como las largas. Además, se vio que la diferencia entre alumnos de alto y bajo rendimiento disminuye en las clases que normalmente emplean más tiempo en los deberes.

Por tanto, hay evidencias de que los deberes escolares se asocian positivamente con el rendimiento académico y, además, parece haber una relación recíproca. Es decir, no solo los deberes escolares benefician el rendimiento académico, sino que también un buen rendimiento académico ayuda en la realización de los deberes escolares. Esto precisamente comprueban algunos estudios como el llevado a cabo por Irene Pan, Bibiana Regueiro, Beatriz Ponte, Susana Rodríguez, Isabel Piñeiro y Antonio Valle (2013), con una muestra de estudiantes de educación primaria. Así, como reveló este estudio, los estudiantes con un mayor rendimiento académico se implican más en los deberes escolares (especialmente en lo que se refiere a la cantidad de deberes realizados y al aprovechamiento del tiempo dedicado a esos deberes).

Objetivos e hipótesis

A partir de estos planteamientos, el principal propósito de este trabajo es analizar las relaciones entre el rendimiento académico en matemáticas de los estudiantes de primaria y los enfoques de trabajo que ponen en marcha al realizar los deberes escolares y su grado de implicación en ellos.

Teniendo en cuenta la investigación previa en relación con los enfoques de aprendizaje, se espera encontrar que aquellos estudiantes con los niveles más altos de rendimiento en matemáticas sean los que adopten más un enfoque profundo y aquellos con menor rendimiento tengan un enfoque superficial al realizar los deberes.

Parece haber una relación positiva entre la cantidad de deberes realizados y el rendimiento académico; por esto, se espera encontrar que aquellos estudiantes con los niveles más altos de rendimiento académico en matemáticas serán los que realicen una mayor cantidad de deberes. Adicionalmente, se espera encontrar que los niveles más altos de rendimiento académico en matemáticas estén asociados con un mejor aprovechamiento del tiempo dedicado a los deberes. En cambio, se espera una relación nula, e incluso negativa, entre el rendimiento académico en matemáticas y el tiempo empleado al realizar los deberes en esta asignatura.

\section{Método}

\section{Participantes}

La muestra está integrada por 897 estudiantes pertenecientes a 13 centros públicos de educación primaria de la provincia de A Coruña (España). El 50,2\% son hombres y el 49,8\% son mujeres, de edades comprendidas entre los 9 y los 13 años ( $M=10.77)$. Del total, 437 de ellos cursaban $5^{\circ}$ curso de educación primaria (224 alumnos y 213 alumnas) y 460 cursaban $6^{\circ}$ curso de educación primaria (227 alumnos y 233 alumnas).

\section{Instrumentos}

Para evaluar los enfoques de trabajo al realizar los deberes se utilizó el Inventario de Procesos de Estudio (IPE), elaborado por Pedro Rosário, José Carlos Núñez y Julio A. González-Pienda (2006). Como paso previo a su aplicación, se hizo un ajuste de los ítems al proceso de realización 
de los deberes escolares en la asignatura de matemáticas. El cuestionario está compuesto por 12 ítems agrupados en dos factores o dimensiones: enfoque superficial y enfoque profundo. Este instrumento está basado en muchas de las aportaciones de las investigaciones más relevantes en el campo de los enfoques de aprendizaje y estudio (Biggs, Kember \& Leung, 2001; Rosário, Núñez, González-Pienda, Almeida, Soares \& Rubio, 2005; Wong, Lin \& Watkins, 1996). Los ítems de este instrumento se presentan en un formato tipo Likert con cinco alternativas de respuesta que van desde 1 (totalmente falso) hasta 5 (totalmente cierto).

Para medir las variables vinculadas con la implicación en los deberes se utilizó la Encuesta sobre los Deberes Escolares (EDE), una escala que evalúa diferentes dimensiones relativas a la eficacia de los deberes para el aprendizaje y rendimiento académico de los alumnos. De hecho, numerosos estudios han utilizado estas dimensiones para evaluar la implicación de los estudiantes en los deberes escolares (Núñez, Suárez, Cerezo, González-Pienda, Rosário, Mourão \& Valle, 2015; Núñez, Suárez, Rosário, Vallejo, Cerezo \& Valle, 2015; Rosário, Mourão, Baldaque, Nunes, Núñez, González-Pienda, Cerezo \& Valle, 2009; Valle, Pan, Núñez, Rosário, Rodríguez \& Regueiro, 2015). En todos los casos, los ítems estaban referidos a los deberes escolares en la asignatura de matemáticas. En concreto, las dimensiones evaluadas son las siguientes:

1. La estimación de la cantidad de deberes realizados por los alumnos se obtuvo mediante las respuestas a un ítem relativo a la cantidad de deberes realizados habitualmente, utilizando para ello una escala tipo Likert con cinco alternativas ( 1 = ninguno, 2 = algunos, 3 = la mitad, 4 = casi todos, 5 = todos).

2. En cuanto al tiempo diario dedicado a la realización de los deberes, los estudiantes respondieron a tres ítems (en general, en una semana típica, en un fin de semana típico) con la formulación general "¿Cuánto tiempo sueles dedicar a la realización de los deberes?", siendo las opciones de respuesta $1=$ menos de 30 minutos, 2 = de 30 minutos a una hora, $3=$ de una hora a hora y media, $4=$ de hora y media a dos horas, $5=$ más de dos horas.

3. Finalmente, el aprovechamiento del tiempo dedicado a los deberes se evaluó por medio de las respuestas a tres ítems (en general, en una semana típica, en un fin de semana típico) en los que se les pedía que indicaran el nivel de aprovechamiento del tiempo dedicado habitualmente a los deberes, utilizando para ello la siguiente escala: 1 = lo desaprovecho totalmente (me distraigo constantemente con cualquier cosa), 2 = lo desaprovecho más de lo que debiera, 3 = regular, $4=10$ aprovecho bastante, $5=10$ aprovecho totalmente (me concentro y hasta terminar, no pienso en otra cosa).

La evaluación del rendimiento académico en matemáticas tuvo en cuenta las calificaciones académicas finales obtenidas en esta asignatura por los alumnos participantes. Concretamente, se han establecido las siguientes calificaciones: insuficiente, suficiente, bien, notable y sobresaliente.

\section{Análisis de datos}

Además de los análisis descriptivos de las variables, se llevó a cabo un Análisis Multivariado de Varianza (MANOVA) para conocer si hay diferencias estadísticamente significativas en los enfoques de trabajo que adoptan los estudiantes (VD) en función de los distintos niveles de rendimiento 
académico (VI). Posteriormente, se realizó otro MANOVA para averiguar si hay diferencias significativas en las variables vinculadas con la implicación en los deberes escolares en función del rendimiento académico de los estudiantes. Para la interpretación de los tamaños del efecto se utilizó el criterio establecido por Jacob Cohen (1988), según el cual, un efecto es pequeño cuando $\eta_{p}{ }^{2}=.01(d=.20)$, el efecto es medio cuando $\eta_{p}{ }^{2}=.059(d=.50)$ y el tamaño del efecto es grande si $\eta_{p}^{2}=.138(d=.80)$.

\section{Resultados}

Análisis descriptivos y correlacionales

El enfoque profundo al realizar los deberes mantiene una relación positiva y significativa con el rendimiento en matemáticas, con el aprovechamiento del tiempo al hacer los deberes y con la cantidad de deberes realizados, y una relación negativa con el enfoque superficial. Por otro lado, el enfoque superficial presenta una relación positiva con el tiempo dedicado a los deberes y una relación negativa con la cantidad de deberes realizados, con el aprovechamiento del tiempo y con el rendimiento académico.

El rendimiento académico en matemáticas se correlaciona de modo positivo y significativo con la cantidad de deberes realizados y con el aprovechamiento del tiempo dedicado a los deberes, pero lo hace negativamente con el tiempo dedicado por los estudiantes a realizar esos deberes.

En resumen, el rendimiento académico en matemáticas es más alto cuanto mayor cantidad de deberes realizan los estudiantes, cuanto más aprovechan el tiempo dedicado a esos deberes y cuanto menos tiempo emplean en hacer esos deberes. Al mismo tiempo, ese rendimiento se incrementa cuanto más utilizan los estudiantes un enfoque profundo y cuanto menos utilizan un enfoque superficial al hacer los deberes escolares (ver tabla 1).

Tabla 1

Medias, desviaciones típicas, asimetría, curtosis y matriz de correlaciones

\begin{tabular}{l|c|c|c|c|c|c}
\hline & $\mathbf{1}$ & $\mathbf{2}$ & $\mathbf{3}$ & $\mathbf{4}$ & $\mathbf{5}$ & $\mathbf{6}$ \\
\hline 1. Enfoque profundo & - & & & & & \\
2. Enfoque superficial & $-.30^{\mathrm{a}}$ & - & & & & \\
3. Cantidad de deberes & $.18^{\mathrm{a}}$ & $-.13^{\mathrm{a}}$ & - & & & \\
4. Tiempo dedicado a los deberes & -.04 & $.10^{\mathrm{a}}$ & .02 & - & & \\
5. Aprovechamiento del tiempo & $.36^{\mathrm{a}}$ & $-.30^{\mathrm{a}}$ & $.25^{\mathrm{a}}$ & $-.10^{\mathrm{a}}$ & - & \\
6. Rendimiento académico & $.13^{\mathrm{a}}$ & $-.22^{\mathrm{a}}$ & $.24^{\mathrm{a}}$ & $-.12^{\mathrm{a}}$ & $.21^{\mathrm{a}}$ & - \\
\hline M & 3.89 & 2.65 & 4.69 & 2.55 & 4.02 & 3.41 \\
DT & 0.76 & 0.75 & 0.68 & 1.07 & 0.84 & 1.27 \\
Asimetría & -0.61 & 0.42 & -2.59 & 0.52 & -0.94 & -0.43 \\
Curtosis & 0.27 & 0.32 & 7.28 & -0.28 & 1.49 & -0.91 \\
\hline \hline
\end{tabular}

Fuente: elaboración propia

${ }^{\mathrm{a}} \mathrm{p}<.01 ;{ }^{\mathrm{b}} \mathrm{p}<.05$.

Rendimiento académico en matemáticas y enfoques de trabajo en los deberes escolares

Los resultados del MANOVA indican que hay diferencias estadísticamente significativas en los enfoques de trabajo tomados conjuntamente en función de los distintos niveles del rendimiento académico $\left(\lambda_{\text {wilks }}=.941\right.$; $\left.F(8,1782)=6.85 ; p<.001 ; \eta_{p}^{2}=.030\right)$ (ver tabla 2$)$. El tamaño del efecto es pequeño. De hecho, la variable independiente solo explica el $3 \%$ de la varianza de la variable dependiente. 
Teniendo en cuenta cada variable dependiente individualmente, los resultados indican que hay diferencias estadísticamente significativas en el enfoque superficial que los estudiantes adoptan al realizar los deberes escolares en función de los distintos niveles de rendimiento académico $\left(F(4,892)=12.34 ; p<.001 ; \eta_{p}^{2}=.052\right)$. El tamaño del efecto es medio. Como se puede observar en la tabla 2 y en el gráfico 1, la adopción de un enfoque superficial a la hora de hacer los deberes escolares parece que va disminuyendo a medida que el rendimiento académico es más alto.

Tabla 2

Estadísticos descriptivos (media, desviación típica) para cada uno de los enfoques de trabajo en función de los niveles del rendimiento académico en matemáticas

Niveles de rendimiento académico en matemáticas

\begin{tabular}{l|c|c|c|c|c|c|c|c|c|c}
\hline & \multicolumn{2}{|c|}{ Insuficiente (1) } & \multicolumn{2}{c|}{ Suficiente (2) } & \multicolumn{2}{c|}{ Bien (3) } & \multicolumn{2}{c|}{ Notable (4) } & \multicolumn{2}{c}{ Sobresaliente (5) } \\
\cline { 2 - 12 } & $M$ & $D T$ & $M$ & $D T$ & $M$ & $D T$ & $M$ & $D T$ & $M$ & $D T$ \\
\hline Enfoque superficial & 2.93 & 0.69 & 2.92 & 0.85 & 2.65 & 0.68 & 2.58 & 0.73 & 2.45 & 0.69 \\
\hline Enfoque profundo & 3.76 & 0.84 & 3.72 & 0.82 & 3.86 & 0.72 & 3.96 & 0.75 & 4.01 & 0.68 \\
\hline \hline
\end{tabular}

Fuente: elaboración propia

También hay diferencias estadísticamente significativas en el enfoque profundo que adoptan los estudiantes al hacer los deberes en función de los distintos niveles de rendimiento académico $(F(4,892)=4.58 ; p<.01$; $\left.\eta_{p}{ }^{2}=.020\right)$. En este caso, el efecto es bastante más pequeño que en el caso del enfoque superficial. De todos modos, la tendencia (tabla 2 y gráfico 1) es que el uso de un enfoque profundo al realizar los deberes escolares se incrementa a medida que es más alto el rendimiento académico.

Gráfico 1

Valores medios en los enfoques de trabajo en los deberes escolares para cada uno de los niveles de rendimiento académico en matemáticas

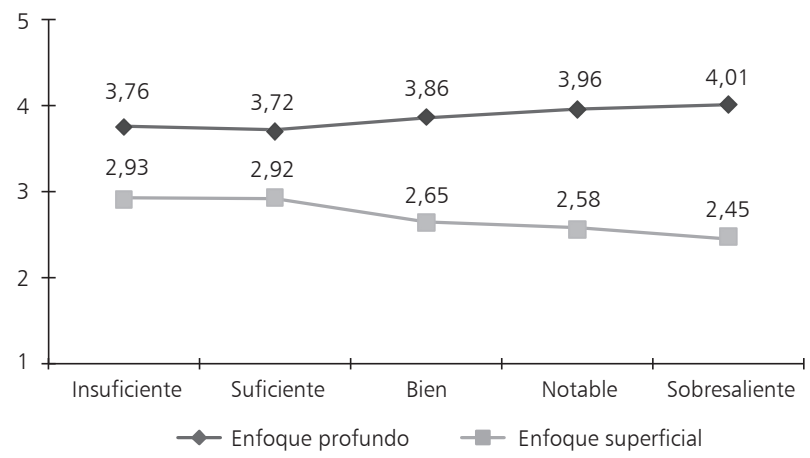

Fuente: elaboración propia

Rendimiento en matemáticas e implicación en los deberes escolares

Los resultados del MANOVA indican que hay diferencias estadísticamente significativas en la implicación en los deberes escolares en función de los distintos niveles del rendimiento académico $\left(\lambda_{\text {wilks }}=.899\right.$; $\left.F(12,2355.01)=8.06 ; p<.001 ; \eta_{p}^{2}=.035\right)$ (tabla 3). El efecto es pequeño. De hecho, la variable independiente solo explica un 3,5\% de la varianza de la variable dependiente. 
En cuanto a los resultados referidos a cada variable dependiente contemplada individualmente, los análisis indican que hay diferencias estadísticamente significativas en la cantidad de deberes realizados de los prescritos en función de los distintos niveles de rendimiento académico $\left(F(4,892)=14.28 ; p<.001 \eta_{p}^{2}=.060\right)$, con un tamaño del efecto medio (tabla 3 y gráfico 2 ).

Tabla 3

Estadísticos descriptivos (media, desviación típica) correspondientes a cada uno de los niveles del rendimiento académico en las variables vinculadas con la implicación en los deberes escolares

\begin{tabular}{|c|c|c|c|c|c|c|c|c|c|c|}
\hline \multicolumn{11}{|c|}{ Niveles de rendimiento académico en matemáticas } \\
\hline & \multicolumn{2}{|c|}{ Insuficiente (1) } & \multicolumn{2}{|c|}{ Suficiente (2) } & \multicolumn{2}{|c|}{ Bien (3) } & \multicolumn{2}{|c|}{ Notable (4) } & \multicolumn{2}{|c|}{ Sobresaliente (5) } \\
\hline & M & $D T$ & M & $D T$ & $M$ & $D T$ & $M$ & $D T$ & $M$ & DT \\
\hline Cantidad de deberes & 4.36 & 1.07 & 4.50 & 0.75 & 4.69 & 0.63 & 4.74 & 0.62 & 4.90 & 0.32 \\
\hline Tiempo de deberes & 2.70 & 1.17 & 2.68 & 1.18 & 2.73 & 1.04 & 2.43 & 1.02 & 2.40 & 0.98 \\
\hline $\begin{array}{l}\text { Aprovechamiento } \\
\text { del tiempo }\end{array}$ & 3.62 & 1.16 & 3.85 & 0.86 & 3.98 & 0.78 & 4.15 & 0.79 & 4.19 & 0.67 \\
\hline
\end{tabular}

Fuente: elaboración propia

En cuanto a los resultados referidos a cada variable dependiente contemplada individualmente, los análisis indican que hay diferencias estadísticamente significativas en la cantidad de deberes realizados de los prescritos en función de los distintos niveles de rendimiento académico $\left(F(4,892)=14.28 ; p<.001 \eta_{p}^{2}=.060\right)$, con un efecto medio (tabla 3 y gráfico 2).

La tendencia que se observa en el gráfico 2 es un aumento progresivo en la cantidad de deberes realizados por los estudiantes, de los prescritos por los profesores, a medida que sus niveles de rendimiento académico son más altos. Y esa cantidad de deberes realizados tiende a ser menor cuanto más bajo es su rendimiento académico.

En cuanto al tiempo dedicado a los deberes escolares, los resultados demuestran que también hay diferencias estadísticamente significativas en función de los distintos niveles de rendimiento académico $(F(4,892)=4.13$; $\left.p<.01 ; \eta_{p}^{2}=.018\right)$, si bien el efecto es pequeño.

\section{Gráfico 2}

Valores medios en las variables vinculadas con la implicación en los deberes escolares para cada uno de los niveles de rendimiento académico en matemáticas

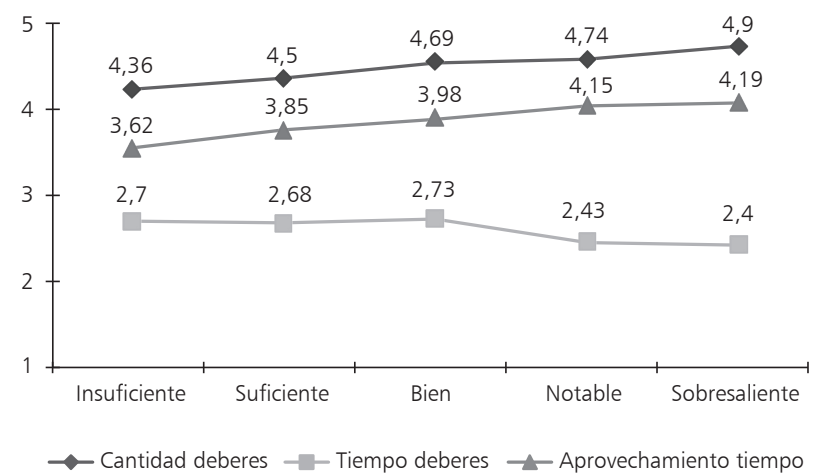

Fuente: elaboración propia 
Aunque estas diferencias no parecen ser tan claras como en el caso de la cantidad de deberes realizados, sí se observa una cierta tendencia que indica que el tiempo que dedican los estudiantes a los deberes parece ser mayor cuando los niveles de rendimiento son más bien bajos o medios. Sin embargo, cuando los niveles de rendimiento son altos o muy altos, el tiempo que dedican a realizar los deberes tiende a disminuir (tabla 3 y gráfico 2).

Por lo que se refiere al aprovechamiento del tiempo dedicado a los deberes, los resultados indican que hay diferencias estadísticamente significativas en esta variable en función de los distintos niveles de rendimiento académico $\left(F(4,892)=10.67 ; p<.001 ; \eta_{p}{ }^{2}=\right.$ .046), con un efecto cercano a medio.

La tendencia de los resultados (tabla 3 y gráfico 2) demuestra que hay un mejor aprovechamiento del tiempo dedicado a realizar los deberes por parte de los estudiantes a medida que sus niveles de rendimiento académico son más altos. Es decir, los estudiantes con los niveles más bajos de rendimiento académico en matemáticas son los que también muestran los niveles más bajos de aprovechamiento del tiempo a la hora de hacer los deberes. Y los que obtienen un rendimiento más alto son también los que aprovechan mejor ese tiempo que dedican a realizar sus deberes escolares.

\section{Discusión}

Los resultados de este estudio indican que los distintos niveles de rendimiento académico que logran los estudiantes en matemáticas están asociados con usos diferentes respecto a los enfoques de trabajo que adoptan al realizar los deberes escolares. Así, según se incrementa el rendimiento académico en matemáticas, hay una mayor utilización de un enfoque profundo y una disminución de un enfoque superficial al realizar los deberes escolares en esta materia.

En cuanto a la implicación en los deberes escolares, los resultados indican que hay un aumento progresivo en la cantidad de deberes realizados y en el aprovechamiento del tiempo dedicado a los deberes, a medida que los niveles de rendimiento académico son más altos. Por otra parte, el tiempo que dedican los estudiantes a realizar los deberes es mayor cuando el rendimiento es bajo o medio. Sin embargo, cuando el rendimiento es alto o muy alto, el tiempo que dedican a realizar los deberes tiende a disminuir. Estos resultados indican que los niveles más altos de rendimiento académico en matemáticas se asocian con una mayor cantidad de deberes realizados y con un mejor aprovechamiento del tiempo dedicado estos deberes.

La mayor parte de las investigaciones muestra una relación positiva entre la cantidad de deberes realizados y el rendimiento académico, en especial en estudiantes de secundaria y bachillerato (Cooper, Robinson \& Patall, 2006), pero también en estudiantes de primaria (Pan, Regueiro, Ponte, Rodríguez, Piñeiro \& Valle, 2013). Con base en estos resultados, se puede afirmar que, de modo general, "hacer los deberes lleva consigo que el rendimiento académico mejore" (Cooper, Robinson \& Patall, 2006, p. 48).

Cooper (1989b) revisó 120 estudios empíricos sobre los efectos de los deberes escolares. Encontró 50 estudios que mostraban correlaciones entre cantidad de tiempo que los estudiantes dedicaban a los deberes y sus logros escolares. En 43 de ellos, los estudiantes que hacían más deberes tenían mejores resultados académicos, mientras que solo 7 de los estudios mostraron relaciones negativas entre ambas variables. Otro aspecto destacado es que para los estudiantes de primaria, la relación entre cantidad de deberes realizados y logros académicos era casi nula. En cambio, esa relación iba siendo cada vez más fuerte y positiva según se iba avanzando hacia secundaria.

En cuanto a las relaciones entre tiempo dedicado a los deberes y rendimiento académico, parece evidente que dedicar demasiado tiempo a los deberes no parece estar relacionado de modo directo y lineal con un buen rendimiento académico. Es más, invertir mucho tiempo puede estar reflejando escasos conocimientos, poca comprensión u otro tipo de problemas como falta de concentración o de motivación. Como indican Ulrich Trautwein y Oliver Lüdtke (2009), es más probable que los tiempos prolongados haciendo los deberes escolares reflejen problemas motivacionales o de comprensión y no que sean un signo de alto esfuerzo y de motivación de los estudiantes. De hecho, el esfuerzo invertido en los deberes escolares ha demostrado tener un impacto más positivo en el rendimiento académico que el tiempo dedicado a los deberes (Schmitz \& Skinner, 1993; Trautwein, Lüdtke, Schnyder \& Niggli, 2006).

Pedro Rosário, Rosa Mourão, José Carlos Núñez, Julio A. González-Pienda y Paula Solano (2006) indican que, en general, hay una relación positiva entre tiempo empleado en los deberes escolares y rendimiento académico en los últimos cursos de secundaria y bachillerato, pero esta relación es poco clara en educación primaria. Añaden, además, que en estudios realizados en otros países, en especial en Estados Unidos, los estudiantes de primaria con bajo rendimiento académico suelen emplear más tiempo en la realización de los deberes escolares, mientras que en los estudiantes de bachillerato la tendencia se invierte, es decir, los de alto rendimiento son los que suelen emplear más tiempo. Por eso, las correlaciones positivas entre tiempo dedicado a los deberes y rendimiento no deben ser contempladas como una evidencia de que por sí solo 
el tiempo empleado conduce necesariamente a un incremento en el rendimiento, ya que es probable que la relación no sea lineal.

\section{Implicaciones educativas}

Los resultados de este trabajo ponen de manifiesto que los niveles más altos de rendimiento en matemáticas están asociados con una mayor utilización de un enfoque de trabajo profundo al realizar los deberes escolares en esta materia. Por el contrario, los niveles más bajos de rendimiento se asocian con una mayor utilización de un enfoque superficial al realizar los deberes escolares.

Al mismo tiempo, los resultados también parecen demostrar que cuanto mayor es el rendimiento en matemáticas también aumentan la cantidad de deberes escolares que realizan los estudiantes y el aprovechamiento del tiempo que dedican a realizar esos deberes. Este resultado parece coincidente con una gran parte de la investigación sobre deberes escolares, pero tiene de novedoso que es con estudiantes de educación primaria, área en la que parece que los resultados en relación con estas variables no eran tan claros.

Sin embargo, esta relación positiva entre el rendimiento académico en matemáticas con la cantidad de deberes y el aprovechamiento del tiempo no parece mantenerse con respecto al tiempo dedicado a los deberes, pues la relación con el rendimiento en matemáticas es negativa. De hecho, la tendencia observada es que un mayor tiempo de dedicación a los deberes se asocia con los peores resultados en matemáticas, mientras que un menor tiempo dedicado a los deberes se relaciona con los niveles más altos de rendimiento en esta materia.

Probablemente, aquellos estudiantes que tienen un rendimiento más alto necesitan menos tiempo, porque tienen más habilidades y conocimientos previos o porque gestionan mejor el tiempo que dedican a los deberes (o ambas cosas a la vez) que aquellos estudiantes que tienen un rendimiento más bajo. Incluso, es posible que cuanto más bajo sea el rendimiento académico también sean menos las habilidades y los conocimientos y haya mayores dificultades para gestionar bien el tiempo dedicado a los deberes (aunque estuvieran motivados para ello). De hecho, en algunos trabajos (p.e., Valle, Pan, Regueiro, Suárez, Tuero \& Nunes, 2015) se ha demostrado que aquellos estudiantes que gestionan bien el tiempo dedicado a los deberes ( $y$ no necesariamente los que dedican más tiempo) son quienes más profundamente trabajan en ellos.

En definitiva, de los resultados se deduce que uno de los caminos para incrementar los niveles de rendimiento en matemáticas de nuestros estudiantes pasa necesariamente por cambios radicales en la ma- nera de enfrentarse a las tareas y aprendizajes de los contenidos de esta materia. Para que esto sea posible, es necesario que los estudiantes con bajos resultados académicos en matemáticas vayan logrando ciertos niveles de éxito en algunas tareas de matemáticas. La labor que debe cumplir el profesorado a la hora de adaptar esas tareas a los niveles de dificultad de cada alumno es absolutamente determinante. Las propias experiencias de éxito no solo van a contribuir a incrementar progresivamente la competencia percibida de estos alumnos sino que deben ayudar también a generar modos o enfoques distintos de enfrentarse a las tareas de matemáticas, pues sentirse más competente se vincula con una intención más clara por aprender y comprender los contenidos matemáticos. Por eso, para mejorar el rendimiento de nuestros estudiantes en matemáticas, resulta clave conseguir que su enfoque de trabajo en esta materia sea cada vez más profundo y menos superficial.

Pero esos niveles de rendimiento en matemáticas también se pueden ver mejorados si la prescripción de deberes escolares tiene en cuenta algunos de los resultados derivados de este trabajo. Los niveles más bajos de rendimiento académico se asocian con una menor cantidad de deberes realizados (de los prescritos por el profesorado de matemáticas), con un menor aprovechamiento del tiempo dedicado a los deberes, e incluso con un mayor tiempo de dedicación a realizar los deberes. Por tanto, realizar mayor cantidad de deberes de los prescritos por el profesorado y aprovechar bien el tiempo dedicado a esos deberes, está relacionado positivamente con buenos resultados académicos en matemáticas.

De este modo, la cantidad de deberes realizados y la gestión del tiempo dedicado a esos deberes son dos variables muy importantes a la hora de establecer posibles pautas para mejorar los resultados de los estudiantes en matemáticas. Probablemente, como plantean Barry J. Zimmerman y Anastasia Kitsantas (2005), aquellos estudiantes que realizan más cantidad de deberes escolares también suelen tener una competencia percibida más alta. Estos autores consideran que la realización exitosa de un mayor número de deberes escolares constituye una variable predictora de la percepción de autoeficacia, ya que está relacionada tanto con la capacidad para aprender como también con la propia responsabilidad en el aprendizaje. De hecho, estos autores sugieren que una vez que los estudiantes realizan los deberes escolares con éxito, esto favorece positivamente su autoeficacia percibida en el ámbito concreto de realización de los deberes. Por tanto, la cantidad de deberes realizados con éxito en matemáticas puede ser, probablemente, una fuente muy poderosa para incrementar las creencias de autoeficacia en esta materia. Si esto se consigue, no solo aumenta 
un pilar motivacional como la autoeficacia percibida (Valle, Regueiro, Rodríguez, Piñeiro, Freire, Ferradás \& Suárez, 2015), sino que los sentimientos negativos asociados a las matemáticas irán decreciendo y dejarán de percibir los contenidos matemáticos como una amenaza y sí como un reto y un desafío para el estudiante.

\section{Agradecimientos}

Este trabajo se ha desarrollado gracias a la financiación del proyecto de investigación EDU2013-44062-P, perteneciente al Plan Estatal de Investigación Científica y Técnica y de Innovación 2013-2016 (Ministerio de Economía, Industria y Competitividad, MINECO) y al financiamiento recibido por una de las autoras en el programa Formación de Profesorado Universitario, FPU del Ministerio de Educación, Cultura y Deporte, MECD.

\section{Sobre los autores}

Antonio Valle - Arias es catedrático de psicología de la educación y director del departa- mento de Psicología Evolutiva y de la Educación, Universidad de A Coruña (España). Es coordinador del grupo de investigación en Psicología Educativa (GIPED), Universi- dad de A Coruña. Sus principales líneas de investigación se centran en la motivación académica, los deberes escolares, las estrategias de estudio y el aprendizaje auto- rregulado. Actualmente, es responsable del proyecto de investigación del MINECO (Ref. proyecto: EDU2013-44062-P) sobre la relación entre los deberes escolares y el rendimiento académico.

Bibiana Regueiro es contratada en el departamento de Psicología Evolutiva y de la Educación, Universidad de A Coruña (España). Es miembro del grupo de investigación en Psicología Educativa (GIPED), Universidad de A Coruña. Sus líneas de investigación se centran en el estudio de la motivación e implicación en los deberes escolares y sus relaciones con el aprendizaje y el rendimiento académico.

Natalia Suárez es profesora asociada en el departamento de Psicología, Universidad de Oviedo (España). Su principal línea de investigación se centra en el estudio de las relaciones entre tareas para casa, implicación familiar y rendimiento académico.

José C. Núñ̃ez es catedrático de dificultades de aprendizaje y director del departamento de Psicología, Universidad de Oviedo (España). Sus principales líneas de investigación son: a) dimensiones psicológicas y educativas del aprendizaje autorregulado en contextos educativos; b) deberes escolares; c) dificultades del aprendizaje escolar y trastorno por déficit de atención e hiperactividad, TDAH. Ha participado en proyectos de investigación de convocatorias autonómicas (Universidad de Oviedo, Fondos regionales), nacionales (Centro de Investigación y Documentación Educativa, CIDE; Ministerio de Ciencia y Tecnología, MCyT; Ministerio de Educación, Cultura y Deporte, MECD) e internacionales (Fondo Nacional de Desarrollo Científico y Tecnológico de Chile, FONDECYT).

Pedro Rosario es profesor titular de psicología de la educación, Universidad de Minho (Portugal). Sus principales líneas de investigación son: a) dimensiones psicológicas y educativas del aprendizaje autorregulado; b) tareas para casa; c) procesos de autorregulación en ambientes de aprendizaje tecnológicos y en pizarras electrónicas. Tiene numerosas publicaciones en su país y en el extranjero. Es el investigador principal del grupo GUIA (www.guia-psiedu.com).

Irene Pan es licenciada en psicopedagogía y miembro del grupo de investigación en Psicología Educativa (GIPED), del departamento de Psicología Evolutiva y de la Educación, Universidad de A Coruña. Su principal línea de investigación se centra en el estudio de las relaciones entre tareas para casa, implicación familiar y rendimiento académico. 


\section{Referencias}

Bembenutty, Héfer \& White, Marie Catherine (2013). Academic Performance and Satisfaction with Homework Completion among College Students. Learning and Individual Differences, 24, 83-88.

Biggs, John B. (1987). Student Approaches to Learning and Studying. Melbourne: Australian Council for Educational Research, ACER.

Biggs, John B. (1988). Assessing Student Approaches to Learning. Australian Psychologist, 23 (2), 197-206.

Biggs, John B. (1993). What do Inventories of Students' Learning Processes Really Measure? A Theoretical Review and Clarification. British Journal of Educational Psychology, 63 (1), 3-19.

Biggs, John B.; Kember, David \& Leung, Doris Y. P. (2001). The Revised TwoFactor Study Process Questionnaire: R-SPQ-2F. British Journal Educational Psychology, 71 (1), 133-149.

Cohen, Jacob (1988). Statistical Power Analysis for the Behavioral Sciences. Hillsdale, New Jersey: Lawrence Erlbaum.

Cooper, Harris (1989a). Homework. White Plains, New York: Longman.

Cooper, Harris (1989b). Synthesis of Research on Homework. Educational Leadership, 47 (3), 85-91. Disponible en: https://pdfs.semanticscho lar.org/479a/d93fad486fde6309637e7334fa91525024da.pdf

Cooper, Harris (2001). The Battle over Homework: Common Ground for Administrators, Teachers and Parents. $2^{\text {nd }}$ ed. Thousand Oaks, California: Sage Publications.

Cooper, Harris; Jackson, Kristina; Nye, Barbara \& Lindsay, James J. (2001). A Model of Homework's Influence on the Performance Evaluations of Elementary School Students. The Journal of Experimental Education, 69 (2), 181-199.

Cooper, Harris; Lindsay, James J.; Nye, Barbara \& Greathouse, Scott (1998). Relationships among Attitudes about Homework, the Amount of Homework Assigned and Completed, and Student Achievement. Journal of Educational Psychology, 90 (1), 70-83.

Cooper, Harris; Robinson, Jorgianne Civey \& Patall, Erika A. (2006). Does Homework Improve Academic Achievement? A Synthesis of Research, 1987-2003. Review of Educational Research, 76 (1), 1-62.

Cooper, Harris \& Valentine, Jeffrey C. (2001). Using Research to Answer Practical Questions about Homework. Educational Psychologist, 36 (3), 143-153.

Corno, Lyn (2000). Looking at Homework Differently. Elementary School Journal, 100 (5), 529-548.

Doménech-Betoret, Fernando \& Gómez-Artiga, Amparo (2011). Relación entre las necesidades psicológicas del estudiante, los enfoques de aprendizaje, las estrategias de evitación y el rendimiento. Electronic Journal of Research in Educational Psychology, 9 (2), 463-496. Disponible en: http://www.redalyc.org/pdf/2931/293122840001.pdf

Epstein, Joyce L. \& Voorhis, Frances L. van (2001). More than Minutes: Teachers' Roles in Designing Homework. Educational Psychology, 36 (3), 181-193.

Foyle, Harvey Charles (1984). The Effects of Preparation and Practice Homework on Student Achievement in Tenth-Grade American History. Unpublished doctoral dissertation, Kansas State University, Manhattan, Kansas.

Foyle, Harvey Charles (1990). Homework and Cooperative Learning: A Classroom Field Experiment. Emporia, Kansas: Emporia State University, 
Faculty Research and Creativity Committee. Disponible en: http://fi les.eric.ed.gov/fulltext/ED350285.pdf

Fredricks, Jennifer A.; Blumenfeld, Phyllis C. \& Paris, Alison H. (2004). School Engagement: Potential of the Concept, State of the Evidence. Review of Educational Research, 74 (1), 59-109. Disponible en: http:// journals.sagepub.com/doi/pdf/10.3102/00346543074001059

International Association for the Evaluation of Educational Achievement, IEA (2011). Trends in International Mathematics and Science Study, TIMSS 2011 International Results in Mathematics. Disponible en: http://timssandpirls.bc.edu/timss2011/international-results-mathe matics.html

Jong, Robert de; Westerhof, Klaas Jan \& Creemers, Bert P. M. (2000). Homework and Student Math Achievement in Junior High Schools. Educational Research and Evaluation, 6 (2), 130-157.

Keith, Timothy Z. (1982). Time Spent on Homework and High School Grades: A Large-Sample Path Analysis. Journal of Educational Psychology, 74 (2), 248-253. Disponible en: https://www.scopus.com/record/ display.uri?eid=2-s2.0-0001758506\&origin $=$ inward\&txGid $=1505 \mathrm{a} 2$ 6409f50c02cee14d512f441247

McGrath, J. Barry (1992). Student and Parental Homework Practices and the Effect of English Homework on Student Test Scores. Dissertation Abstracts International, 53 (10A), 3490. (UMI No. 9231359).

Ministerio de Educación, Cultura y Deporte, MECD (2013). PISA 2012. Informe español. Volumen 1. Resultados y contexto. Disponible en: http:// www.mecd.gob.es/inee/Ultimos informes/PISA-2012.html

Muhlenbruck, Laura; Cooper, Harris; Nye, Barbara \& Lindsay, James J. (2000). Homework and Achievement: Explaining the Different Strengths of Relation at the Elementary and Secondary School Levels. Social Psychology of Education, 3 (4), 295-317.

Núñez, José Carlos (2012). Qué sabemos de la relación entre los deberes escolares y rendimiento académico. Algunos datos para el debate. Revista Profesional de la Asociación Nacional de Profesores del Estado, 550, 20-21. Disponible en: http://www.anpe.es/wp-content/ revista/ANPE_550.pdf

Núñez, José Carlos; Suárez, Natalia; Cerezo, Rebeca; González-Pienda, Julio A.; Rosário, Pedro; Mourão, Rosa \& Valle, Antonio (2015). Homework and Academic Achievement across Spanish Compulsory Education. Educational Psychology, 35 (6), 726-746. http://dx.doi.org/10.1080/0 1443410.2013.817537

Núñez, José Carlos; Suárez, Natalia; Rosário, Pedro; Vallejo, Guillermo; Cerezo, Rebeca \& Valle, Antonio (2015). Teachers' Feedback on Homework, Homework-Related Behaviors and Academic Achievement. Journal of Educational Research, 108 (3), 204-216. http://dx.doi.org/ 10.1080/00220671.2013.878298

Núñez, José Carlos; Vallejo, Guillermo; Rosário, Pedro; Tuero, Ellián \& Valle, Antonio (2014). Variables del estudiante, del profesor y del contexto en la predicción del rendimiento académico en Biología: análisis desde una perspectiva multinivel. Revista de Psicodidáctica, 19 (1), 145-172. Disponible en: http://www.redalyc.org/articulo. oa?id=17529569007

Olympia, Daniel E.; Sheridan, Susan M.; Jenson, William R. \& Andrews, Debra (1994). Using Student-Managed Interventions to Increase Homework Completion and Accuracy. Educational Psychology Papers and Publications. Journal of Applied Behavior Analysis, 27 (1), 85-99. 
Disponible en: http://digitalcommons.unl.edu/cgi/viewcontent.cgi?ar ticle $=1035 \&$ context $=$ edpsychpapers

Pan, Irene; Regueiro, Bibiana; Ponte, Beatriz; Rodríguez, Susana; Piñeiro, Isabel \& Valle, Antonio (2013). Motivación, implicación en los deberes escolares y rendimiento académico. Aula Abierta, 41 (3), 13-22. Disponible en: https://dialnet.unirioja.es/descarga/articulo/4401103.pdf

Regueiro, Bibiana; Pan, Irene; Valle, Antonio; Núñez, José Carlos; Suárez, Natalia \& Rosário, Pedro (2014). Motivación e implicación en los deberes escolares: diferencias en función del rendimiento académico y del curso. International Journal of Developmental and Educational Psychology, Revista INFAD de Psicología, 7 (1), 425-436. Disponible en: http://www.infad.eu/RevistaINFAD/OJS/index.php/IJODAEP/arti cle/view/812/752

Regueiro, Bibiana; Rodríguez, Susana; Piñeiro, Isabel; Estévez, Iris; Ferradás, Mar \& Suárez, Natalia (2015). Diferencias en la percepción de la implicación parental en los deberes escolares en función del nivel de motivación de los estudiantes. European Journal of Investigation in Health, Psychology and Education, 5 (3), 313-323. Disponible en: https://dialnet.unirioja.es/descarga/articulo/5388949.pdf

Rosário, Pedro; González-Pienda, Julio A.; Cerezo, Rebeca; Pinto, Ricardo; Ferreira, Pedro; Abilio, Lourenço \& Paiva, Olimpia (2010). Eficacia del programa «(Des)venturas de Testas» para la promoción de un enfoque profundo de estudio. Psicothema, 22 (4), 828-834. Disponible en: http://www.psicothema.com/pdf/3808.pdf

Rosário, Pedro; Mourão, Rosa; Baldaque, Margarida; Nunes, Tánia; Núñez, José Carlos; González-Pienda, Julio A.; Cerezo, Rebeca \& Valle, Antonio (2009). Tareas para casa, autorregulación del aprendizaje y rendimiento en matemáticas. Revista de Psicodidáctica, 14(2), 179-192. Disponible en: http://www.ehu.eus/ojs/index.php/psicodidactica/arti cle/view/721/600

Rosário, Pedro; Mourão, Rosa; Núñez, José Carlos; González-Pienda, Julio A. \& Solano, Paula (2006). Escuela-Familia: ¿Es posible una relación recíproca y positiva? Papeles del Psicólogo, 27 (3), 171-179. Disponible en: http://www.papelesdelpsicologo.es/pdf/1374.pdf

Rosário, Pedro; Núñez, José Carlos; Ferrando, Pere Joan; Paiva, M. Olimpia; Lourenço, Abilio; Cerezo, Rebeca \& Valle, Antonio (2013). The Relationship between Approaches to Teaching and Approaches to Studying: A Two-Level Structural Equation Model for Biology Achievement in High School. Metacognition and Learning, 8 (1), 47-77. Disponible en: https://www.researchgate.net/publication/236233159_The_re lationship_between_approaches_to_teaching_and_approaches_ to_studying_A_two-level_structural_equation_model_for_biology_ achievement_in_high_school

Rosário, Pedro; Núñez, José Carlos \& González-Pienda, Julio A. (2006). Comprometer-se com o estudar na Universidade: Cartas do Gervásio ao seu Umbigo. Coimbra: Almedina.

Rosário, Pedro; Núñez, José Carlos; González-Pienda, Julio A.; Almeida, Leandro S.; Soares, Serafim \& Rubio, Marta (2005). El aprendizaje escolar examinado desde la perspectiva del Modelo 3P de J. Biggs. Psicothema, 17 (1), 20-30. Disponible en: http://www.psicothema. com/pdf/3059.pdf

Schmitz, Bernhard \& Skinner, Ellen (1993). Perceived Control, Effort, and Academic Performance: Interindividual, Intraindividual, and Multivariate Time-Series Analyses. Journal of Personality and Social Psychology, 64 (6), 1010-1028. Disponible en: https://www.researchgate. 
net/publication/232544440_Perceived_Control_Effort_and_Acade mic_Performance_Interindividual_Intraindividual_and_Multivaria te_Time-Series_Analyses

Tam, Vicky C. W. (2009). Homework Involvement among Hong Kong Primary School Students. Asia Pacific Journal of Education, 29 (2), 213227. http://dx.doi.org/10.1080/02188790902859004

Trautwein, Ulrich (2007). The Homework-Achievement Relation Reconsidered: Differentiating Homework Time, Homework Frequency, and Homework Effort. Learning and Instruction, 17 (3), 372-388.

Trautwein, Ulrich; Köller, Olaf; Schmitz, Bernhard \& Baumert, Jürgen (2002). Do Homework Assignments Enhance Achievement? A Multilevel Analysis in Seventh Grade Mathematics. Contemporary Educational Psychology, 27 (1), 26-50.

Trautwein, Ulrich \& Lüdtke, Oliver (2009). Predicting Homework Motivation and Homework Effort in Six School Subjects: The Role of Person and Family Characteristics, Classroom Factors and School Track. Learning and Instruction, 19, 243-258.

Trautwein, Ulrich; Lüdtke, Oliver; Kastens, Claudia \& Köller, Olaf (2006). Effort on Homework in Grades 5-9: Development, Motivational Antecedents, and the Association with Effort on Classwork. Child Development, 77 (4), 1094-1111.

Trautwein, Ulrich; Lüdtke, Oliver; Schnyder, Inge \& Niggli, Alois (2006). Predicting Homework Effort: Support for a Domain-Specific, Multilevel Homework Model. Journal of Educational Psychology, 98 (2), 438-456. Disponible en: https://tu-dresden.de/mn/psychologie/ lehrlern/ressourcen/dateien/lehre/lehramt/lehrveranstaltungen/an gewandte-lernpsychologie/dateien/trautwein_hausaufgabenmodell. pdf?lang $=$ de

Trautwein, Ulrich; Schnyder, Inge; Niggli, Alois; Neumann, Marko \& Lüdtke, Oliver (2009). Chameleon Effects in Homework Research: The Homework-Achievement Association Depends on the Measures Used and the Level of Analysis Chosen. Contemporary Educational Psychology, 34 (1), 77-88.

Valle, Antonio; Pan, Irene; Núñez, José Carlos; Rosário, Pedro; Rodríguez, Susana \& Regueiro, Bibiana (2015). Deberes escolares y rendimiento académico en Educación Primaria. Anales de Psicología, 31 (2), 562569. Disponible en: http://revistas.um.es/analesps/article/view/anale sps.31.2.171131/175611

Valle, Antonio; Pan, Irene; Regueiro, Bibiana; Suárez, Natalia; Tuero, Ellián \& Nunes, Ana Rita (2015). Predicting Approach to Homework in Primary School Students. Psicothema, 27 (4), 334-340. Disponible en: http://www.psicothema.com/pdf/4271.pdf

Valle, Antonio; Regueiro, Bibiana; Estévez, Iris; Piñeiro, Isabel; Rodríguez, Susana \& Freire, Carlos (2015). Implicación y motivación hacia los deberes escolares en los estudiantes de Primaria según el rendimiento académico y el curso. European Journal of Investigation in Health, Psychology and Education, 5 (3), 345-355.

Valle, Antonio; Regueiro, Bibiana; Rodríguez, Susana; Piñeiro, Isabel; Freire, Carlos; Ferradás, Mar \& Suárez, Natalia (2015). Perfiles motivacionales como combinación de expectativas de autoeficacia y metas académicas en estudiantes universitarios. European Journal of Education and Psychology, 8 (1), 1-8. Disponible en: https://www.researchgate. net/publication/281062964_Perfiles_motivacionales_como_com binacion_de_expectativas_de_autoeficacia_y_metas_academicas_ en_estudiantes_universitarios 
Warton, Pamela M. (2001). The Forgotten Voices in Homework: Views of Students. Educational Psychologist, 36 (3), 155-165. http://dx.doi. org/10.1207/S15326985EP3603_2

White, Marie Catherine (2011). Predicting Success in Teacher Certification Testing: The Role of Academic Help-Seeking. The International Journal of Educational and Psychological Assessment, 7 (1), 22-44. Disponible en: https://www.researchgate.net/publication/256459358_Pre dicting_Success_in_Teacher_Certification_Testing_The_Role_of_ Academic Help-Seeking

Woitschach, Pamela; Fernández-Alonso, Rubén; Martínez-Arias, Rosario \& Muñiz, José (2017). Influencia de los centros escolares sobre el rendimiento académico en Latinoamérica. Revista de Psicología y Educación, 12 (2), 138-154. Disponible en: http://www.revistadepsi cologiayeducacion.es/pdf/152.pdf

Wong, Ngai-Ying; Lin, Wen-Ying \& Watkins, David (1996). Cross-Cultural Validation of Models of Approaches to Learning: An Application of Confirmatory Factor Analysis. Educational Psychology, 16 (3), 317-327.

Xu, Jianzhong \& Wu, Hongyun (2013). Self-Regulation of Homework Behavior: Homework Management at the Secondary School Level. The Journal of Educational Research, 106 (6), 1-13. Disponible en: http:// hanoverhslibrary.weebly.com/uploads/1/2/3/3/12336552/self_regu lation_of_homework_behavior_1.pdf

Zimmerman, Barry J. \& Kitsantas, Anastasia (2005). Homework Practices and Academic Achievement: The Mediating Role of Self-Efficacy and Perceived Responsibility Beliefs. Contemporary Educational Psychology, 30 (4), 397-417. Disponible en: http://hanoverhslibrary.weebly. com/uploads/1/2/3/3/12336552/homework_practices_and_acade mic_1.pdf 